\title{
Interaction of cognitive and sensorimotor maps of visual space
}

\author{
BRUCE BRIDGEMAN and SHELLEY PEERY \\ University of Califormia, Santa Cruz, Califormia \\ and \\ SULEKHA ANAND \\ Stanford University School of Medicine, Stanford, California
}

\begin{abstract}
Studies of saccadic suppression and induced motion have suggested separate representations of visual space for perception and visually guided behavior. Because these methods required stimulus motion, subjects might have confounded motion and position. We separated cognitive and sensorimotor maps without motion of target, background, or eye, with an "induced Roelofs effect": a target inside an off-center frame appears biased opposite the direction of the frame. A frame displayed to the left of a subject's center line, for example, will make a target inside the frame appear farther to the right than its actual position. The effect always influences perception, but in half of our subjects it did not influence pointing. Cognitive and sensorimotor maps interacted when the motor response was delayed; all subjects now showed a Roelofs effect for pointing, suggesting that the motor system was being fed from the biased cognitive map. A second experiment showed similar results when subjects made an open-ended cognitive response instead of a five-alternative forced choice. Experiment 3 showed that the results were not due to shifts in subjects' perception of the felt straight-ahead position. In Experiment 4, subjects pointed to the target and judged its location on the same trial. Both measures showed a Roelofs effect, indicating that each trial was treated as a single event and that the cognitive representation was accessed to localize this event in both response modes.
\end{abstract}

Several topographic maps represent the visual world in the cortex (Felleman \& Van Essen, 1991). This characteristic of the visual system raises a question for visual physiology: do all of these maps work together to create a single representation of visual space, or are they functionally distinct? If they are distinct, how many functional maps are there and how do they communicate with one another? This paper presents psychophysical evidence for at least two functionally distinct representations of the visual world in normal humans; under some conditions, the two representations can simultaneously hold different spatial values. The paper also demonstrates some of the ways in which the representations communicate with one another.

An early hint that cognitive and sensorimotor systems are separable in normal humans came from studies of eye movements. On the one hand, subjects are unaware of sizable displacements of the visual world if they occur during saccadic eye movements, implying that information about spatial location is degraded during saccades (Bridgeman, Hendry, \& Stark, 1975; Brune \& Lücking, 1969; Ditchburn, 1955; Mack, 1970; Wallach \& Lewis, 1965).

On the other hand, people do not become disoriented after saccades, implying that spatial information is main-

\footnotetext{
A preliminary report of Experiment 1 appeared as "Complementary Cognitive and Motor Image Processing" in G. Obrecht \& L. Stark (Eds.), Presbyopia Research, New York: Plenum Press, 1990. Correspondence concerning the present article should be addressed to B. Bridgeman, Department of Psychology, University of California, Santa Cruz, CA 95064 (e-mail: bruce@cats.ucsc.edu).
}

tained. Some experimental evidence supports this conclusion, though there is disagreement in the literature and many points remain controversial. For instance, an early report that the eyes can saccade accurately to a target that is flashed (and mislocalized) during an earlier saccade (Hallett \& Lightstone, 1976) has been criticized because there were only a few target positions, which subjects might have memorized. Others have failed to replicate the result (Dassonville, Schlag, \& Schlag-Rey, 1992; Honda, 1990). Still others have found accurate pointing to targets flashed during saccades, however (Hansen, 1979; Hansen \& Skavenski, 1977), and hand-eye coordination remains fairly accurate following saccades (Festinger \& Cannon, 1965; Honda, 1985). It would seem in the balance that under at least some conditions, accurate behavior can be maintained. How can loss of perceptual information and maintenance of visually guided behavior exist side by side?

To resolve this discrepancy, we noted that the conflicting observations were made with different response measures. The experiments on saccadic suppression of displacement require a nonspatial verbal report or buttonpress, both symbolic responses. Successful orienting of the eye or hand, in contrast, requires quantitative spatial information with a one-to-one correspondence between stimulus position and motor output. The conflict between experiments involving symbolic responses and those involving motor responses might be resolved if the two types of report, which have been labeled "cognitive" and "sensorimotor" 
(Paillard, 1987), could be combined in a single experiment (Bridgeman, Lewis, Heit, \& Nagle, 1979). The saccadic suppression of displacement experiments addresses only the cognitive system. It is at the cognitive level that symbolic decisions such as button pressing or verbal response are mediated. If two pathways in the visual system process different kinds of information, spatially oriented motor activities might have access to accurate position information even when that information is unavailable at a cognitive level. The cognitive and sensorimotor systems correspond, respectively, to the representational and sensorimotor modes of Paillard (1991), who presents other evidence for a separation of the two modes. An assumption that one system is egocentric and the other exocentric has been suggested (Paillard, 1987), but it is not a necessary part of the hypothesis.

The two conflicting observations, saccadic suppression on the one hand and accurate motor behavior on the other, have been combined by asking subjects to point to the position of a target that had been displaced and then extinguished (Bridgeman et al., 1979; Prablanc, Echallier, Komilis, \& Jeannerod, 1979). Subjects were also asked whether or not the target had been displaced. Extinguishing the target and preventing the subjects from viewing their hands (open-loop pointing) guaranteed that only internally stored spatial information could be used for pointing. The displacement was detected on some trials, whereas on others it went undetected, but pointing accuracy was similar whether or not the displacement was detected in the cognitive system.

This result implied that control of motor activity was unaffected by the perceptual detectability of target position. Alternatively, it is possible to interpret the result in terms of signal detection theory, as reflecting a high response criterion for the report of displacement. That is, a target displacement could go unreported by cognitive measures because of a high criterion or threshold for reporting displacements, even while the displacement affected spatial behavior when a choice of a direction was compulsory. This possibility was tested with a twoalternative forced-choice cognitive measure of saccadic suppression of displacement (Bridgeman \& Stark, 1979). This criterion-free measure showed no information about displacement to be available to the cognitive system when pointing was affected.

A more rigorous method of separating cognitive and motor systems is to introduce a signal only into the motor system in one condition and only into the cognitive system in another. We know that induced motion affects the cognitive system because we experience the effect and because subjects can make verbal judgments of it. But the experiments above imply that the information used for pointing might come from sources unavailable to perception.

We inserted a signal selectivity into the cognitive system with stroboscopic induced motion (Bridgeman, Kirch, \& Sperling, 1981). A frame surrounding a fixed target was displaced, creating the illusion that the target had jumped in the opposite direction. Target and frame were then extinguished, and the subject pointed open-loop to the last position of the target. Trials on which the target had seemed to move to the left were compared with trials on which it had seemed to move to the right. Pointing was not significantly different in the two kinds of trials, showing that the induced motion illusion did not affect pointing. In a second condition, information was inserted selectively into the motor system by nulling the cognitive signal. Each subject adjusted the real motion of the target, which jumped in phase with the frame, until the target seemed stationary. Thus, the cognitive system specified a stable target. Nevertheless, subjects pointed in significantly different directions when the target was extinguished in the left or the right position, showing that the difference in real target positions was still available to the motor system.

This result suggests that the motor system detected a target displacement that was not available to the cognitive system. Thus a double dissociation occurred: in the first condition, the target displacement affected only the cognitive measure, and in the second, it affected only the motor behavior. Alternatively, the cognitive system could have ascribed the visually specified displacement to an artifact of frame movement.

Cognitive and sensorimotor functions have also been dissociated by feeding the two systems opposite signals at the same time. The experiment again involved stroboscopic induced motion; a target jumped in the same direction as a frame, but not far enough to cancel the induced motion (Wong \& Mack, 1981). Although the target still appeared to jump in the direction opposite the frame, it actually jumped in the same direction. Saccadic eye movements followed the veridical direction even though subjects perceived stroboscopic motion in the opposite direction. If a delay in responding was required, however, eye movements followed the perceptual illusion, implying that the motor system has a short-lived memory and must rely on information from the cognitive system if response is delayed.

Some evidence has appeared recently that requires a reexamination of these results (Brenner \& Smeets, 1994; Smeets \& Brenner, 1995). Using careful psychophysics, the work seems to show that a moving background influences perceptual judgment of a target's velocity, but not of its position, implying that all of the studies cited above suffered from a confounding of velocity and position estimates that was misinterpreted as requiring two visual systems. In fact, the data show just the opposite, that motion of a background has similar effects on perception of motion and of position. As in the studies cited above, the essential difference was between perception and action, not between motion and position.

The crucial condition involves ocular tracking of a target against a background that could move either in the same direction as the target, or in the opposite direction. In different trials, the target's perceived speed and its perceived final position were measured. In this situation, one obtains an induced motion: the target appears to move faster if the background moves in the opposite direction, or more slowly if the background moves in the same direction. Does the induced motion affect only motion, as the authors contend, or both motion and position? 
For the position judgment, the observer saw the target move against the background for a variable interval, after which the target disappeared. The task was to place another target where the original target had been at the time of its disappearance. The result was that the positioning of the second target seemed unaffected by the direction of background motion. The task was not performed immediately, however: the background continued to move for $500 \mathrm{msec}$ after the original target's disappearance, and this movement is the critical problem. When the target is on the screen, it is seen against a large background pattern. At the time of its disappearance, the target coincides with some texture element that continues to move across the screen. If we make the almost inescapable assumption that observers judge the position of the target relative to the texture that surrounded it, and not relative to some distant peripheral frame or to an abstract egocentric direction, then the result has nothing to do with differentiation between velocity and position, but only with the delay between the disappearance of the background and the time of response. Coincidentally, the duration of the delay in background disappearance was precisely of the appropriate length to obliterate the effect of induced motion on the position judgment. The position judgment appeared to be unaffected by the background velocity only because the drift from induced motion was compensated by an equal and opposite drift during the delay before the judgment was made.

Another way of thinking about the result is in terms of eye movements. The observer's eyes must involuntarily track the texture element that last overlapped the target--in fact, it is nearly impossible not to track a large texture as it drifts across a screen. The background moved at $6 \mathrm{~cm} / \mathrm{sec}$, so that in $500 \mathrm{msec}$ it moved $3 \mathrm{~cm}$ beyond its position when the target disappeared. The target would be carried $3 \mathrm{~cm}$ during this interval, or back to the position it would have occupied had the induced motion had no effect. This is precisely the size of the observed effect. When the target moves from left to right and the background moves in the opposite direction, for example, the target appears to move rightward faster than it otherwise would. After the target is extinguished, the background carries the eye and the texture previously superimposed with the target back to the left again (Figure 1). The illusion is created, and then destroyed, before the subject responds.

Velocity matching in the Brenner and Smeets studies (Brenner \& Smeets, 1994; Smeets \& Brenner, 1995) did not show this effect because the background was static, with no period of texture visibility between exposure to the standard target and exposure to the comparison target whose velocity was matched.

Another recent study of the effects of moving backgrounds on several response measures compared ocular and manual tracking (Masson, Proteau, \& Mestre, 1995). The conditions most relevant to the present issues are those in which a background moved either with or against the direction of a target motion, while the subject tracked the target with either pursuit eye movements or continu- ous manual tracking. Manual tracking followed the perceptual effects of induced motion, with faster tracking when the target was moving against the background direction, and seemed to be moving faster. Oculomotor tracking followed an opposite pattern, with faster tracking when the background and target moved in the same direction. Thus the subjects perceived the target as moving more slowly, but tracked more quickly. The total tracking pattern remained accurate, however, for the ocular tracking was closed-loop and small error accumulations were compensated by saccades.

Masson et al. (1995) interpret their ocular tracking results in terms of retinal slip, with the resulting retinal motion driving the arm's angular velocity. As the background changes from moving against the target to moving with the target, for example, the velocity of retinal slip decreases and the arm velocity decreases also. Another way to interpret this result is to do so in terms of two oculomotor control systems: an optokinetic system that reflexively tracks the entire visual array, and a phylogenetically newer system controlling foveal pursuit (Bridgeman, 1986; Post \& Leibowitz, 1985). The moving background stimulates the older system, so that pursuit must add more innervation to maintain eye velocity when the background moves against the target than when it moves with the target. The pursuit gain is not enough to completely compensate for the changes in optokinetic stimulation from background motion, so the eye shows a velocity bias for the direction of background motion relative to the target. Pursuit gain is higher when the background moves opposite the target, because the pursuit system must work harder to overcome the opposite optokinetic innervation. This higher gain is then used to control the manual system. No "modulated, background-velocity dependent, target retinal velocity signal" (Masson et al., 1995, p. 851) need be integrated with oculomotor efference copy, for the signal is already there in the pursuit system. Since the pursuit innervation is perceived as target motion, while the optokinetic innervation is not perceived, the perceptual induced motion is explained with the same signals. As a result, manual tracking matches perception more closely than oculomotor tracking does; but the subject does not perceive the discrepancy, because low-level dynamic motor signals control the quantitative details of the behavior.

All of these experiments involved stimulus motion or displacement, and they reveal the complexity of the dynamics of several distinct control systems and their realtime interactions. They leave open the possibility that the dissociations between cognitive and motor responses are related in some way to motion systems rather than to representation of visual space per se (as in both Smeets \& Brenner, 1995, and Abrams \& Landgraf, 1990, note). A new method, reported in this paper, can test dissociations of cognitive and motor function without any motion or displacement of the eye or the stimuli at any time during a trial. The dissociation is based on the Roelofs effect (Roelofs, 1935), a tendency to misperceive the position of the edge of a large target presented in an unstructured field. 


\section{Direction of Motion}

Opposite

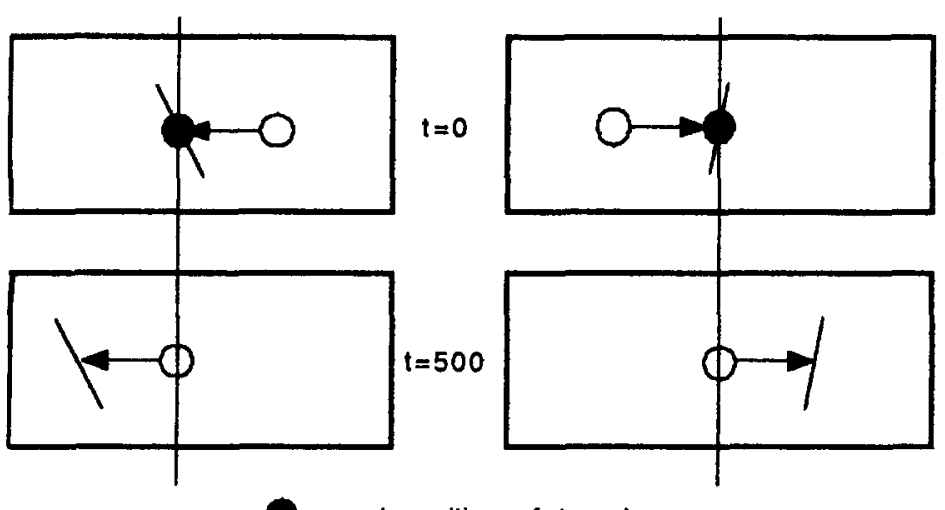

: real position of target

Same

\section{O : apparent position of target}

The effect has also been observed as a tendency to perceive the locations of light flashes as closer to the line of sight than their true positions (Mateeff \& Gourevich, 1983).

Here we elaborate on the principle of the Roelofs effect in order to measure the misperception of target position in the presence of a surrounding frame presented asymmetrically in the field; this is an "induced Roelofs effect," but it will be called a Roelofs effect below. Locations of targets within the frame tend to be misperceived in the direction opposite the offset of the frame. The effect is similar to stroboscopic induced motion in which only the final positions of the target and frame are presented (Bridgeman \& Klassen, 1983).

\section{EXPERIMENT 1}

\section{Method}

\section{Subjects}

The 10 subjects were 9 undergraduate volunteers and the first author. All had normal or corrected-to-normal visual acuity.

\section{Apparatus}

Subjects sat with heads stabilized before a hemicylindrical vertical screen that provided a homogeneous field of view $180^{\circ}$ wide $\times 50^{\circ}$ high. A rectangular frame $21^{\circ}$ wide $\times 8.5^{\circ}$ high $\times 1^{\circ}$ in line width was projected, via a galvanic mirror, and was centered on the subject's midline, or $5^{\circ}$ left, or $5^{\circ}$ right of center. Inside the frame, an " $x " 0.35^{\circ}$ in diameter could be projected via a second galvanic mirror in one of five positions, $2^{\circ}$ apart, with the middle " $x$ " on the subject's midline (Figure 2).

Motor responses were recorded using an unseen pointer with its axis attached to a potentiometer mounted near the center of curvature of the screen and its tip near the screen (Foley \& Held, 1972). A simple analog circuit gave a voltage proportional to the tip's position. The voltage was fed into the A/D converter of a computer that controlled trial presentation and data collection. Pointing data were recorded on a continuous scale. Between trials, subjects moved the pointer to its rightmost position, where it could be found by touch for the next trial. Subjects held the pointer with the forefinger on its tip, so that their arms were outstretched and most of the rotation came from the shoulder. Cognitive responses, or perceived target positions, were recorded from a keyboard placed in front of the subject. There were five keys corresponding to the five target positions; on pointing trials, an "enter" key was pressed to indicate that the pointer was in the desired position.

\section{Procedure}

Training. The subjects were first shown the five possible target positions in sequence, repeated three times, on an otherwise blank screen. Each target was visible for $1 \mathrm{sec}$; the next target followed after a blank interval of $0.5 \mathrm{sec}$. Then the subjects underwent a se- 


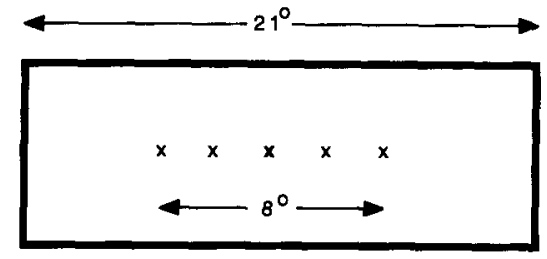

$\mathbf{x}$

$\mathbf{x}$

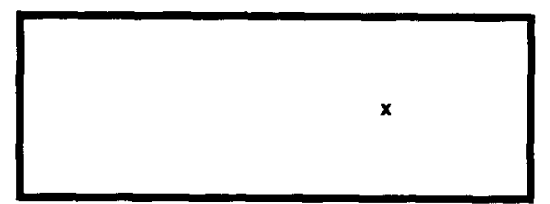

Figure 2. Stimulus array used in Experiments 1 and 2. The frame could be centered (top), offset $5^{\circ}$ right (middle), or offset $5^{\circ}$ left (bottom). A target appeared in one of the five positions indicated within the top frame. Targets always appeared in the same positions, regardless of frame position, and only one target was visible at a time. The center target (shown in boldface in the top frame) is shown in the frames offset left and right. Only one target and frame were shown in a given trial.

ries of training trials. Each trial began with a randomly selected target exposed for $1 \mathrm{sec}$. The subjects estimated the target position with one of the five response keys ( judging trials), and continued with feedback until they were correct on five consecutive trials. Next, they were trained on pointing, without feedback, with the same stimuli in a new random sequence (pointing trials), until they spontaneously returned the pointer to its rightmost position (as initially instructed) on five consecutive trials. Pointing required less practice because there were no positions to memorize; the procedure served only to familiarize the subjects with the apparatus.

In both conditions, the subjects were instructed to wait until the offset of the stimulus before responding. Presenting the target alone forced the subjects to use an egocentric judgment, and the long display time reduced the possibility of target onset eliciting a spurious neural motion signal that might bias responses.

No-delay condition. The 30 types of trials ( 5 target positions $\times$ 3 frame positions $\times 2$ response modes) were mixed in a pseudorandom order. On each trial, one of the five targets and one of the three frames were presented with simultaneous onset, exposed for $1 \mathrm{sec}$, and simultaneously extinguished. The subjects were instructed to fixate the target, in accord with their spontaneous tendency. Because they could not respond until the offset of the stimuli, they were looking at a blank field at the time of the response. Thus the task was a response to an internally stored representation of the stimulus, not a perceptual task.

The pseudorandom trial order was restricted so that pointing trials and judging trials with the same target and frame positions would alternate in the series. After a given target and frame position were presented in a pointing trial, for example, a judging trial would be presented the next time that target and frame combination appeared in the random sequence. Thus, two successive identical trials could not occur in the series. Judging and pointing trials appeared in a random sequence, not alternating. Each trial type was repeated five times, for a total of 150 trials. At stimulus offset, sub- jects heard a short "beep" tone to indicate a judging trial, or a longer "squawk" tone to indicate a pointing trial. There was a rest period after every 50 trials. Responses were not speeded in either trial type, and response latencies were not recorded.

Trials were collated and stored by the computer. Two separate two-way analyses of variance (ANOVAs) were run for each subject and each response mode (assessing target main effect, frame main effect, and interaction). One ANOVA was based on the raw data, and the other on errors (deviations of responses from the actual stimulus positions). The subject with the minimum $F$ is reported below for each condition; $F$ s for other subjects were equally significant or more significant. In general, descriptive statistics are given in the figures and inferential statistics in the text.

Delay condition. Procedures were the same as in the no-delay condition, except that a delay was interposed between stimulus offset and the tone that indicated the type of response that the subject was to make (judging or pointing). Thus, the subjects did not know during the delay interval which type of response would be required.

\section{Results}

\section{No-Delay Condition}

Cognitive measure. In the analyses of the raw data, all 10 subjects showed a significant main effect of target position in both trial types. This means that when the target was toward the left the subjects judged or pointed significantly further to the left than when the target was on the right.

There was also a significant main effect of frame position in judging trials $[F(2,4)>61.2, p<.001]$, for all subjects]. Thus, all subjects showed a Roelofs effect (Figure 3), a tendency to judge the target to be farther to the left than its actual position when the frame was on the right, and vice versa. The mean magnitude of the Roelofs effect was a difference of $2.0^{\circ}$ between judgments with the frame on the left and judgments with the frame on the right. This is a reliable effect; it was present in all subjects under all conditions (Figure 4) and was statistically significant for all subjects. There were no significant interactions, either here or in the subsequent data.

Analyses of the errors in judging target position showed a significant effect of actual target position for all 10 subjects $[F(2,4)>61.2, p<.001]$, with the range of position estimates being smaller than the range of target positions. For the pointing measure, there was a tendency in the opposite direction: 6 subjects showed significant overreaching of the target eccentricities, 1 showed significant underreaching, and 3 had no statistically significant errors. The difference in slopes could yield substantial differences in localization for the pointing and judging modes under some conditions, especially when the frame was offset and the target was at one of its extreme positions. Under these conditions, differences between judging and pointing to the same target in the same trial block could amount to more than $5^{\circ}$.

Motor measure. The main effect of frame position in pointing trials yielded a sharp division of the subjects into two groups: 5 subjects showed a highly significant Roelofs effect $[F(2,4)>18, p<.01]$, whereas the other 5 showed no sign of an effect $[F(2,4)<3.16, p>.18]$ (see Figure 5). The bimodal distribution shows that two qual- 


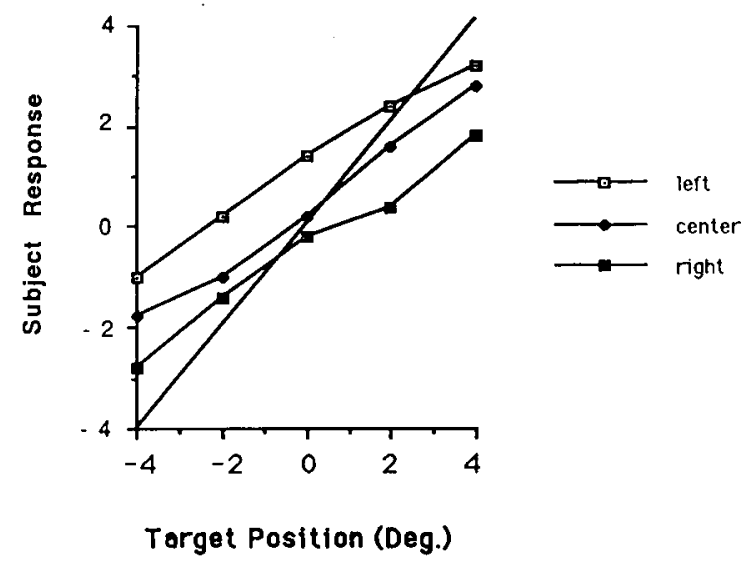

Figure 3. Five-alternative forced-choice judging of target position immediately after stimulus offset; average from 10 subjects. The separation of the three curves corresponding to the three frame positions labeled on the right is due to the Roelofs effect. The diagonal line in this and subsequent figures indicates veridical performance. For this and subsequent figures, the vertical axis is subject response on the 1-5 scale, converted to degrees of angle with 0 as straight-ahead, negative left. Magnitude of the Roelofs effect is given in the text.

itatively different results were obtained; the distribution of significances between subjects was not due to a small, normally distributed effect being significant for some subjects and not for others. That is, each subject showed either a large, robust effect or no sign of a frame influence. Thus, responses on pointing trials were qualitatively different from responses on judging trials for half of the subjects; these subjects showed a Roelofs effect only for judging. The differences in performance among subjects were unrelated to their degree of psychophysical experience or knowledge of the experiment.

Figure 6 illustrates the pointing behavior of 1 subject (A) who showed no Roelofs effect in pointing (right side of Figure 5), and 1 (B) who showed a significant Roelofs effect (left side of Figure 5).

\section{Delay Condition}

Cognitive measure. Nine of the 10 subjects were also tested with a 4-sec delay interposed between display offset and tone (Figure 7). Eight of the 9 showed a significant Roelofs effect for the judging task $[F(2,4)>18, p<$ .01 ], with a mean difference of $2.12^{\circ}$ between perceptual localization when the frame was on the left and when it was on the right. In other words, the localization due to difference in frame position changed by a little more than one target position. This result is comparable to the result in the no-delay condition; results in that condition for the 9 subjects run in the delayed condition were indistinguishable from the data reported above. Subject A's results are presented in Figure 8.

Motor measure. The major difference between the results in this condition and in the no-delay condition was that 7 of the 9 subjects showed a significant Roelofs effect for the pointing task $[F(2,4)>6.94, p<.05$ for 2 subjects; $F(2,4)>18, p<.01$ for 7 subjects].

One of the 2 remaining subjects showed no significant effect of frame position for either measure. The other subject whose pointing behavior still showed no effect of the frame (Figure 9) was retested with an 8-sec delay between display offset and tone. A Roelofs effect was found both for judging trials $[F(2,4)>61.2, p<.001]$ (Figure 10 ) and for pointing trials $[F(2,4)>61.2, p<.001]$ (Figure 11), indicating that after an 8-sec delay this subject's pattern of responses corresponded to that of the majority of subjects at the 4-sec delay.

Again the analysis of response error showed significant tendencies to underestimate the eccentricities of peripheral targets in the judging task for all subjects $[F(2,4)>$ $61.2, p<.001]$, while eccentricity in the pointing task tended to be overestimated. This is shown by slopes of less than 1 in the graphs of judging, and slopes of more than 1 in the graphs of pointing. Five subjects showed significant overreaching in the pointing task, 3 showed slopes not significantly different from the stimulus slope, and 1 showed significant underreaching. This result can be sum-

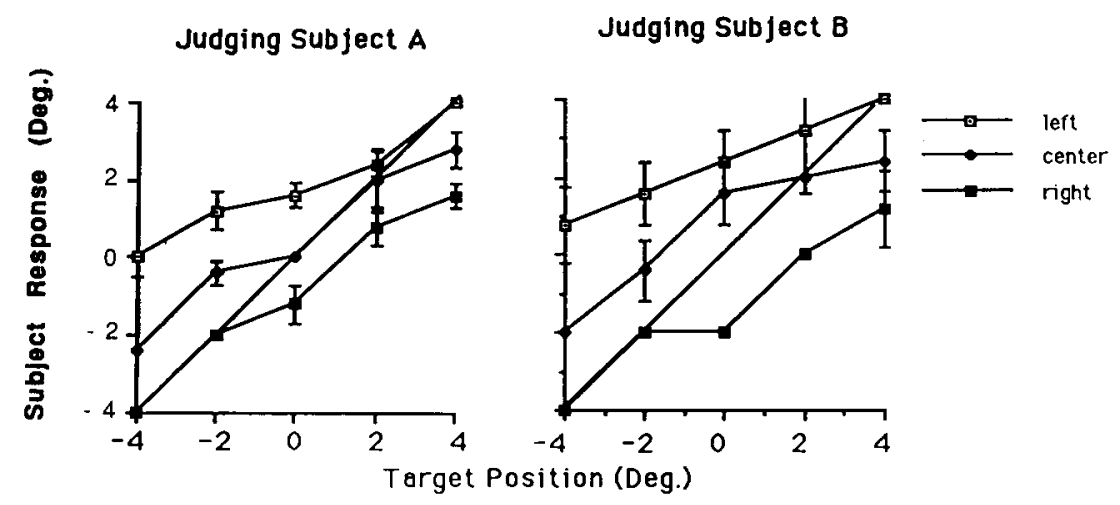

Figure 4. Judging of target position immediately after stimulus offset. Data illustrated here are from 2 subjects, though statistical analyses in the text are from all subjects. The similar behavior of these subjects in judging contrasts with their different behavior in the pointing task (Figure 6). Lack of error bars indicates that the standard deviation was less than the width of the symbol. Display format is as in Figure 3. 


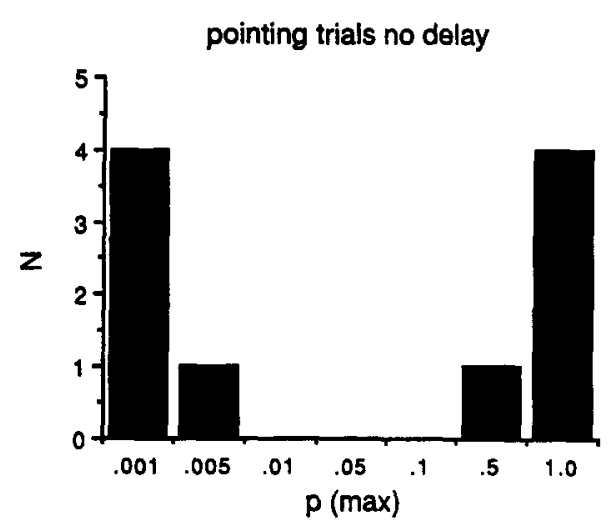

Figure 5. Statistical significance of within-subjects two-way ANOVA frame (Roelofs) effects. Each bin contains subjects with results at least as significant as the probability indicated on the abscissa, but not as significant as the next probability to the left. The bimodal distribution shows two different types of subjects, widely spaced, with no intermediate cases.

marized as a compression of perceived space in the judging mode, with an $8^{\circ}$ field represented by estimates less than $8^{\circ}$ apart. The same $8^{\circ}$ range in the pointing mode, with the same stimuli, usually resulted in pointer positions more than $8^{\circ}$ apart.

\section{Discussion}

Interposing a long enough delay before the response cue forces all subjects to use pointing information that is biased by the frame position, even though half of the subjects were not vulnerable to this bias when responding immediately. This result implies that the bimodal distribution of Roelofs effects in pointing without delay had been due to the subjects using two different sources of information for pointing. One group had used a motor mode that was not sensitive to visual context, a "privileged loop" between vision and motor activity. The other group imported spatial information from the cognitive system into the sensorimotor representation for use in pointing and imported the illusion along with it.

In addition to differences in the Roelofs effect, the results show a differential decay rate of cognitive responses and pointing responses, along with a steeper slope and greater variability for pointing than for cognitive measures. An interpretation that is consistent with cortical neurophysiology, as well as with the literature cited in the introduction, is that the two measures access information from different maps of visual space. The two-maps hypothesis also provides a parsimonious language for describing the results. The motor representation is accessed by a pointing measure that requires an isomorphic response, a 1:1 relationship between stimulus position and behavior; stimulus and response map directly onto one another. The cognitive representation, in contrast, requires a categorization in which the relationship between target position and behavior is arbitrary.

While there were substantial individual differences in the Roelofs effects in pointing, each subject showed a greater slope with pointing than with judging measures. The result cannot be interpreted by resorting to the fact that judging is less accurate than pointing, because this interpretation would predict shallower slopes of the pointing functions due to statistical regression to the mean. The pattern of results was the reverse of this, however;

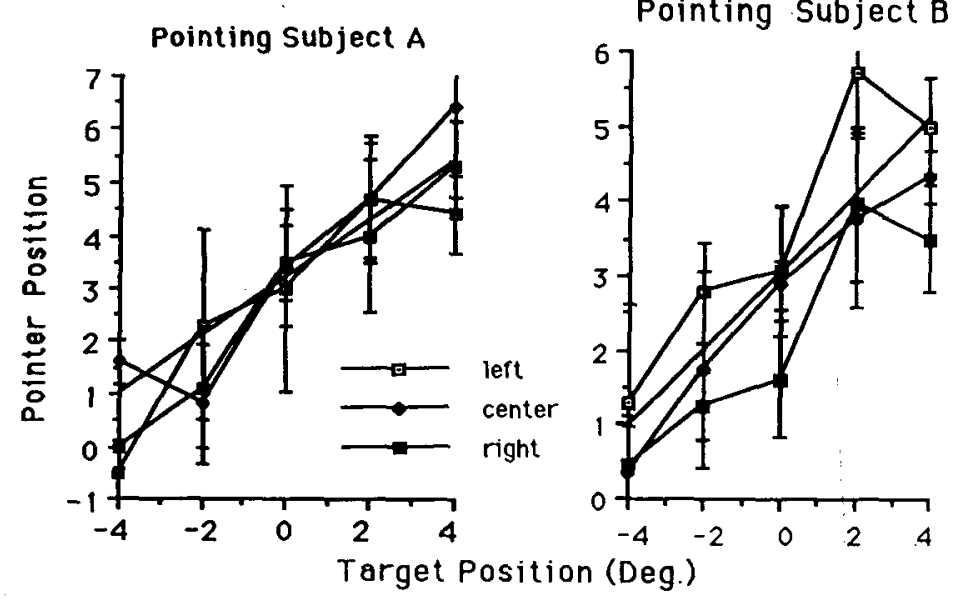

Figure 6. Pointing to targets under the same perceptual conditions as in Figure 3 , in trials randomly intermingled with the judging trials. Subjects $A$ and B correspond to the 2 subjects in Figure 3. 0verlap of the three curves for Subject $A$ indicates lack of influence of frame position on pointing behavior. Separation of the curves for Subject $B$ indicates a continuing influence of the frame. Roelofs effect magnitude for Subject $B$ is $1.4^{\circ}$. All subjects showed either the Type A or the Type B pattern. 


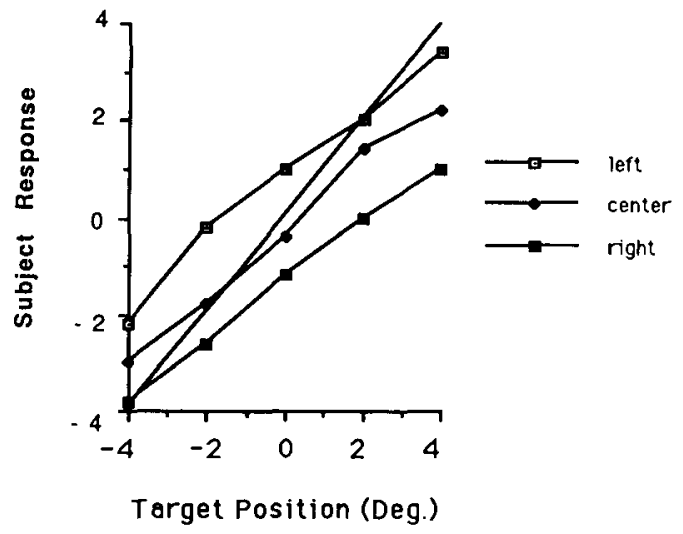

Figure 7. Judging of target position after a 4-sec delay. Data are averaged from 9 subjects. Display format is as in Figure 3.

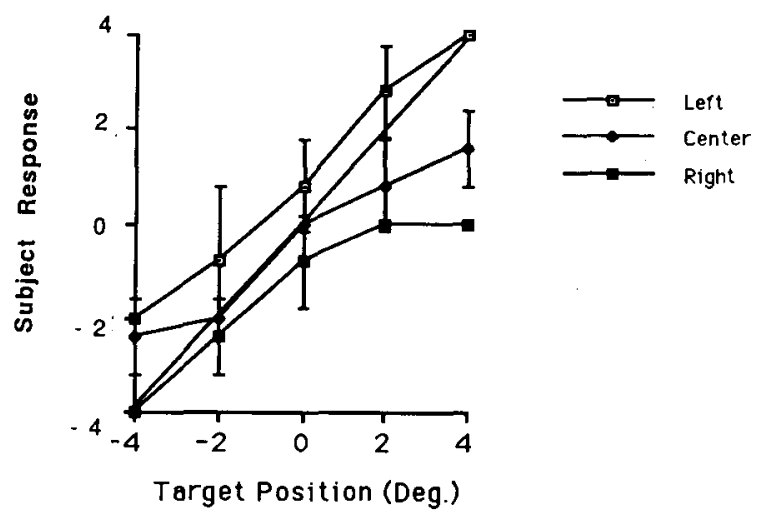

Figure 8. Judging of target position after a 4-sec delay for Subject $A$. Results are similar to those in Figure 3, showing a Roelofs effect of $2.3^{\circ}$. Display format is as in Figure 3 .

the steeper slope (in pointing) was associated with the larger variability. Another possible explanation for the slope difference was sought in Experiment 2.

Another possible explanation of the lower slope with the judging measure is not that space became compressed, but that the interpretation of the remembered target locations became expanded. By using a different kind of scale for judging, we also tested this possibility in Experiment 2.

\section{EXPERIMENT 2}

The subjects in Experiment 1 were free to point in directions lying on a continuum and extending beyond the actual locations of the most extreme targets on either side. Judging, in contrast, was restricted to five discrete locations. The subjects were forced to remain within the $8^{\circ}$ range of the target stimuli. It is possible that the restriction in judging trials may have influenced the observed differences between the pattern of results in judging and pointing trials, and may have forced the observed compression of visual space in the judging mode. To investi- gate this possibility, a new set of subjects was tested with a continuous measure of judged target direction.

\section{Method}

\section{Subjects}

Five new subjects were run, 3 of them experienced psychophysical observers. All were naive about the purposes of the experiment.

\section{Apparatus and Procedure}

The procedure was the same as that in Experiment 1, except for the measure of judgment of target direction in the judging trials. The subjects were not told that there were only five possible target positions. Instead, they were shown a centimeter ruler on the screen during an orientation session before the trials began, and they were asked to estimate the position of an " $x$ " to be displayed on each trial in centimeters to the left or right of the center. Fractions of centimeters could be used, though in practice the subjects seldom used them. Response was always cued immediately after stimulus offset. Pointing trials exactly like those of Experiment 1 were interleaved with the judging trials as before.

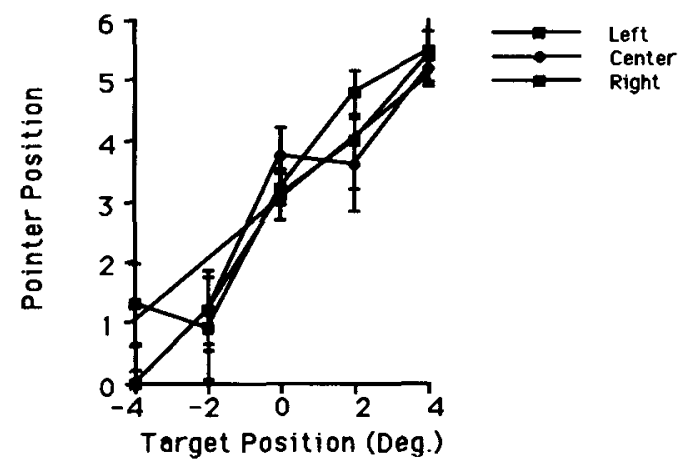

Figure 9. Pointing to targets after a 4-sec delay for Subject $A$, from trials intermingled with those shown in Figure 7. For this subject, no Roelofs effect is evident for pointing; the other subjects showed an effect at this delay. Display format is as in Figure 6.

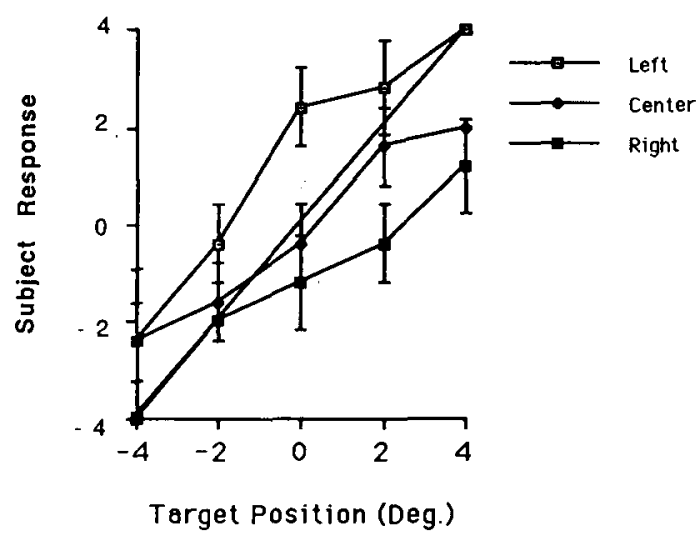

Figure 10. Judging of target position after an 8-sec delay, for Subject $A$. Results are similar to those in Figures 3 (no delay) and 7 (4-sec delay), showing a stable representation of visual positions in the system controlling target position judgments. Display format is as in Figure 3. Roelofs effect is $2.2^{\circ}$. 


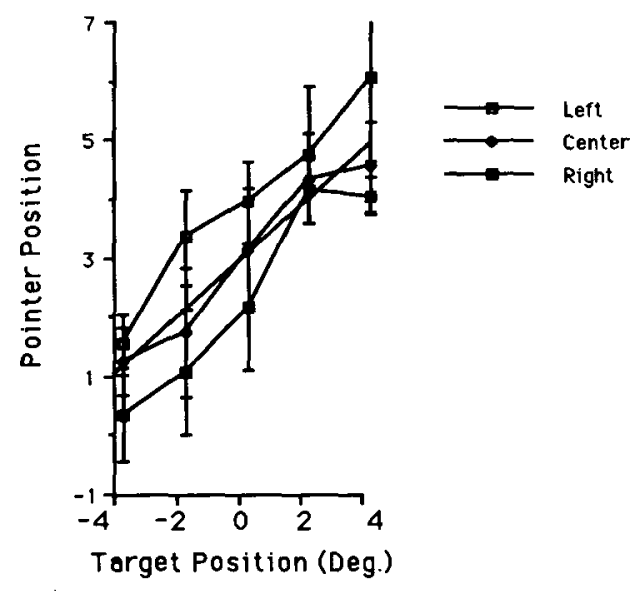

Figure 11. Pointing to targets after an 8-sec delay for Subject $A$. A $1.9^{\circ}$ Roelofs effect for pointing has appeared, indicating that frame position affects pointing just as it affects judging at this latency, though the effects of a separate spatial representation are still evident: slopes of the lines here are more than 1 , in contrast to slopes of less than 1 at all latencies for judging. Display format is as in Figure 6.

\section{Results}

The pattern of results was similar to that of the results from Experiment 1, despite the different response measure for judging. All subjects showed an effect of frame position in judging, although this effect was statistically less significant for 2 of the subjects $(p=.064$ and $p=.068$ ). The result is illustrated in Figure 12. The pointing results were indistinguishable from the pointing results of Experiment 1 (which is not surprising, since the conditions were exactly replicated). In the pointing measure, the subjects again fell into two discontinuous groups, one with significant Roelofs effects $(p<.027, n=2)$ and the other with no Roelofs effects $(p>.124, n=3)$.

In judging, variability was somewhat greater with the centimeter estimation method, resulting in a statistically less reliable Roelofs effect for 2 of the subjects $(p=.064$ and .068). The pattern of results was always in the direction of a Roelofs effect, with perceived position biased to the side opposite the frame position, however, and the absolute magnitudes of the Roelofs effects in Experiments 1 and 2 were similar. When asked how many target positions had been presented, the subjects were uncertain but generally guessed six or seven rather than the five actually given.

The slopes of the lines describing verbal estimate versus target position in Figure 12 are less than 1, in 4 of the 5 subjects, while slopes in the pointing trials (not illustrated) continued to be more than 1 , showing that the compression of visual space found in Experiment 1 was not due to the restricted range of alternative responses (Figure 13). Rather, it seems to represent a more general difference between the internal representations of "cognitive" and "sensorimotor" visual space.

\section{Discussion}

In Experiments 1 and 2, we tested 15 subjects. All of them showed a Roelofs effect in the cognitive measure, showing that the effect, though quantitatively small, is robust and easily obtained. Seven of these subjects showed a Roelofs effect in pointing without a delay, whereas 8 did not. All those tested with delays showed a pointing Roelofs effect after a sufficient delay.

The results of Experiment 2 showed a compression of space comparable to that found in Experiment 1, despite the use $c_{f} d$ different response measure. It is unlikely that the result was due to an expansion of the remembered scale, for the scale in Experiment 1 was learned point by point, whereas that in Experiment 2 was seen all at once as a unit. A similar expansion of both types of scale would be a remarkable coincidence. The apparent compression in Experiment 1 was not due to a reluctance by the subjects to use the extremes of the scale, however, because the same compression occurred with a more open scale in Experiment 2.

These results contrast with those of Bacon, Gordon, and Schulman (1982), who found a Roelofs effect in a comparable paradigm. In one of their conditions, a target oscillated vertically while a background frame oscillated horizontally, resulting in an induced slant in the target's trajectory. Alternatively, the background could be fixed at either its left or its right extreme position, and the target, at either its highest or its lowest point. Pointing to the target yielded a significant horizontal target offset in trials when the frame was on the right as opposed to trials when it was on the left, a replication of the Roelofs effect. The effect was significantly smaller than perceptual effects in an induced-motion control, however, indicating again that the pointing measure is significantly closer

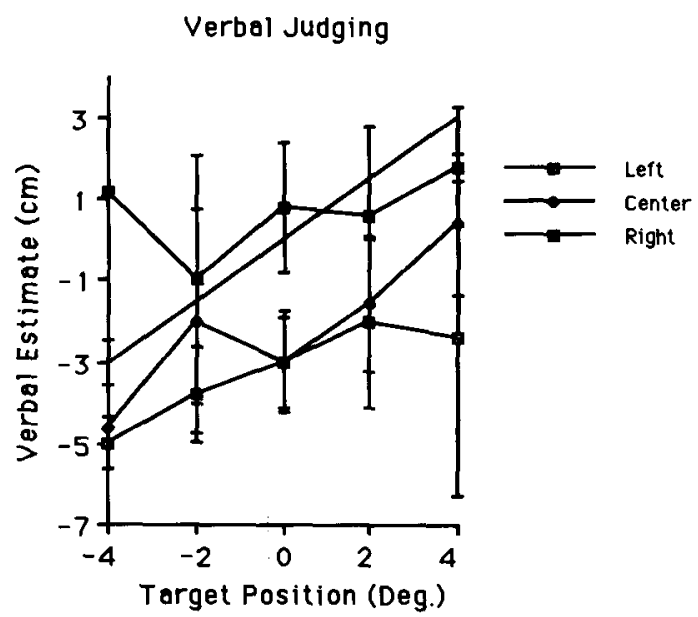

Figure 12. Judging of target position with no delay, using an estimate of target position in centimeters left or right of the center. The results are more variable but otherwise similar to those of Experiment 1; there is a compression of visual space represented in slopes of less than 1 , despite the lack of range restriction in the response mode. A Roelofs effect of $2.0 \mathrm{~cm}$ is also evident. Data are from 1 subject. 


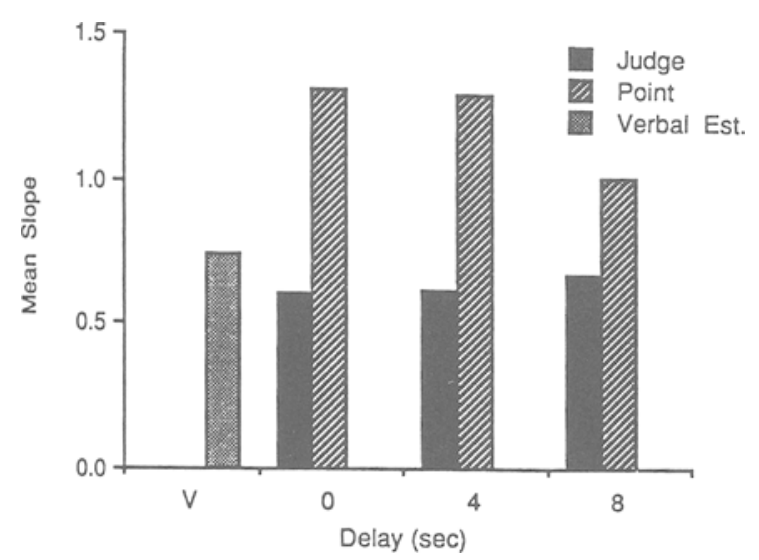

Figure 13. Slopes of the regression lines of target position versus judged position or pointer position for trials with free verbal response (left bar) or five-alternative forced-choice pointing (right bars). Space is compressed in the judging mode even with free response.

to veridical than the perceptual measure. The implicit slant between the target's two positions in the Roelofs condition was $30^{\circ}$; the veridical slant was $45^{\circ}$. Neither they nor we measured response latency, and unspeeded pointing cued by target offset was used in both experiments. But if Bacon et al.'s subjects were not quick about their pointing, a situation similar to our delay condition could have occurred, with a similar result. A similar set of circumstances might account for the similar results of Sugarman and Cohen (1968). We found that most subjects showed a Roelofs effect with a 4-sec delay, but we cannot exclude the possibility that the minimum delay is much shorter; Bacon et al.'s results suggest that it is indeed shorter.

Some of the variability in judging in Experiment 2 probably arose from the subjects' uncertainty about the number of centimeters to assign to a perceived position; several subjects noted this after the conclusion of the experiment. High variability would tend to reduce the statistical significance of the results. Another possible source of variability is a change in the felt position of the straightahead direction on the screen, as a function of frame position. This possibility was investigated in Experiment 3.

\section{EXPERIMENT 3}

There is a possible interpretation of these results that does not require an assumption of separate topographic representations for cognitive and motor functions. According to this interpretation, the biased frame induces a change in a subject's perceived orientation (I. Howard, personal communication, May 2, 1991). Cognitive judgments are then made relative to the false orientation, resulting in a Roelofs effect. For example, a target presented at the center may be judged to be to the left when the frame is projected to the right, because the subject perceives the body's centerline to be to the right of its true position. The centered target is then to the left of the per- ceived centerline. Pointing remains accurate because the body in fact remains in the same position, so that the illusion of orientation does not affect the position of the arm when pointing. As a result, only judging shows a Roelofs effect. Pointing to the centerline of the apparatus, however, should be biased because of the change in egocentric position information. Brosgole (1968) found a shift in perceived straight-ahead in the presence of an eccentric frame.

This hypothesis generates predictions that can be tested empirically. First, it predicts that pointing should never show a Roelofs effect, though the subjects in Experiments 1 and 2 showed such an effect when the response was delayed. Second, the appearance of the pointing Roelofs effect was not accompanied by any change in the judging behavior. This observation contradicts the egocentric illusion hypothesis, because any changes in perceived straight-ahead after the delay should affect both judging and pointing, but effects were seen only in pointing. One could even argue that any changes in perceived straightahead should preferentially influence judging, by the line of reasoning given above, but our result was the reversedelay affected only pointing. Finally, the hypothesis predicts that the felt position of the center of the apparatus should be biased by the presence of an off-center frame. This prediction was tested by having subjects point to the center of the apparatus, which was located on their own midline, while the frame was projected in the same three positions as were used in the previous experiments. This mini-experiment was designed as a test of possible egocentric position illusions, even though the evidence cited above argues against their existence.

\section{Method}

\section{Subjects}

Three subjects, all experienced psychophysical observers, participated in this experiment. Two of them had been subjects in Experiment 2, and 1 had been a subject in Experiment 1 . All had shown a Roelofs effect for pointing.

\section{Apparatus and Procedure}

Using the same unseen pointer as in the above experiments, the subjects adjusted the pointer so that its tip fell in the median plane of the apparatus, defined as halfway between the (invisible) left and right edges of the screen. This was equivalent to pointing straight ahead, because the subjects knew that they were physically located with the center of the apparatus straight ahead. After pressing a key to record the estimate in the computer, the subject swung the pointer to the side for the next trial. The frame was projected for $1 \mathrm{sec}$ in its left, center, or right positions. Pointing took place immediately after the target was extinguished. The target spot was not presented in this experiment. Each subject gave 25 pointing responses for each of the three frame positions, in a random order.

\section{Results}

All of the subjects showed the same pattern of behavior. None showed a significant change in pointer position as a function of frame position [minimum $F(2,4)<$ $2, p>.56]$. Thus, the position of the frame had no effect on the subjects' estimates of the center, nor did they re- 
port any change in felt orientation relative to the center. They always perceived the center of the screen and their own median planes to be congruent.

\section{Discussion}

These results can be applied in two ways to the judging and to the pointing results of Experiments 1 and 2 . All of the subjects had previously shown significant Roelofs effects in judging the target position. Thus the Roelofs effects observed previously with the cognitive measure could not be due to shifts of felt position. Further, to explain the Roelofs effects observed in all subjects for delayed pointing in Experiment 1, one would have to postulate that the biased frame had no effect on felt position immediately after stimulus offset, but that somehow it began to affect pointing after a 4-sec delay. This interpretation is at best implausible.

Brosgole (1968) found a shift in perceived straightahead with an eccentric frame, and Werner, Wapner, and Bruell (1953) found a shift in pointing to straight-ahead under similar conditions. In the present experiments, fixing the head in the straight-ahead position might have been enough to prevent these effects.

\section{EXPERIMENT 4}

The first experiment showed that the motor map is short lived; after a delay, subjects relied on the cognitive map even during motor tasks. In Experiment 4, we asked whether either response modality could affect the other modality when both types of responses were performed on each trial. In other words, we examined whether use of the accurate motor representation of space on a trial would eliminate the Roelofs effect in the cognitive (judging) task, or alternatively whether accessing the cognitive representation would induce a Roelofs effect in the motor measure.

\section{Method}

\section{Subjects}

The subjects were 9 undergraduate volunteers and 1 graduate volunteer. All of the subjects were naive with respect to the purpose of the experiment.

\section{Apparatus and Procedure}

The procedure was the same as that for Experiment 1, except that subjects both pointed to and judged the target location on every trial. Half of the subjects were randomly assigned to point first and half to judge first on each trial. Responses were not delayed. Each combination of the three frame positions and five target locations was repeated 10 times, yielding a total of 150 trials for each subject.

\section{Cognitive Measure}

\section{Results}

Again, all subjects showed the same pattern of results. ANOVAs showed that target location had a significant effect of amplitude $2.2^{\circ}$ for all subjects, whether they judged first $[F(2,4)>16.35, p<.015$; Figure 14] or pointed first $[F(2,4)>17.15, p<.013]$.

\section{Motor Measure}

The pattern of results was similar to that in the cognitive measure. Frame location again had a significant effect of amplitude $4.4^{\circ}$ for all subjects, whether they judged first $[F(2,4)>8.74, p<.037$; Figure 15] or pointed first $[F(2,4)>7.08, p<.05]$. Overall, the average magnitude of the target effect was larger for the pointing responses than for the judging responses $[F(1,1350)=378.5, p<$ $.0001]$. That is, as in Experiment 1, there were steeper slopes for pointing responses than for judging responses.

\section{Discussion}

In contrast to the situation in which subjects either point to or judge the location of a target surrounded by an offset frame with no delay between stimulus presentation and response, when both responses occur to a single stimulus they are both biased by a Roelofs effect. This effect is independent of the order in which the responses occur, suggesting that a separate map is not accessed for each response. That is, instead of activating cognitive and motor maps on each trial for the judging and pointing responses respectively, only one representation of visual space is accessed on each trial. This representation appears to be the cognitive map, suggested by the reliable manifestation of a Roelofs effect in the judging responses of Experiments 1 and 2. Experiment 1 showed the motor map to be short lived, forcing subjects to rely on the cognitive representation when there was a delay before responding. Experiment 4 elaborated on the temporal sequence from cognitive to sensorimotor processing of visual space by showing that motor responses depend on the cognitive map when one can anticipate that such a map will be used for a given visual stimulus.

If the motor system normally is insensitive to stimulusbased biases such as the Roelofs effect, why did subjects in Experiment 4 show a Roelofs effect during pointing? Our interpretation is that the combined sequential re-

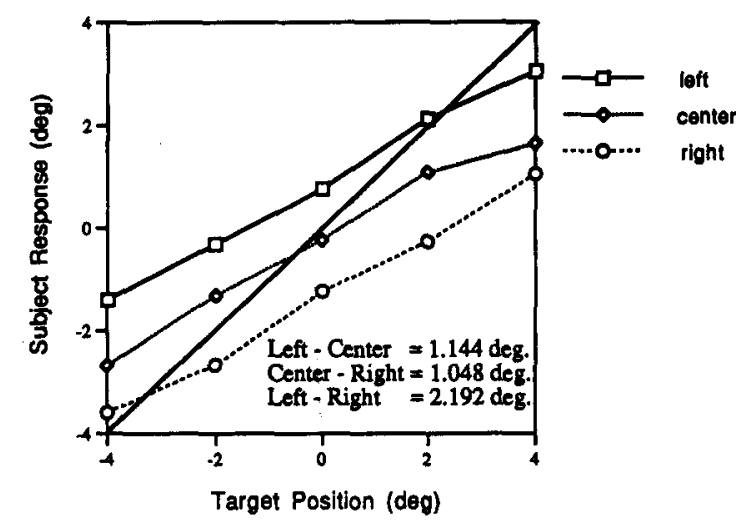

Figure 14. Cognitive (judging) responses of subjects who judged target location and then pointed to target location following stimulus offset on each trial. Data were nearly identical for subjects who pointed first in each trial. Solid lines are least squares regression lines through each function. Display format is as in Figure 3. 


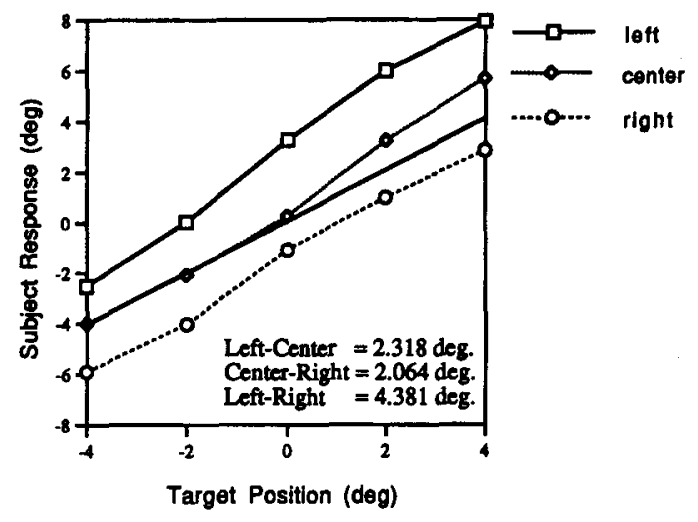

Figure 15. Motor (pointing) responses of subjects who judged target location and then pointed to target location following stimulus offset on each trial. Data were nearly identical for subjects who pointed first in each trial. Solid lines are least squares regression lines through each function.

sponses of pointing and judging required several seconds, and the subjects' knowledge that the position would have to be remembered during that time forced its storage in the cognitive representation, even when the motor response came first. Each trial was treated as a visual "event," encoded in one map of visual space. Since the dual response took more time than the motor representation could support, responses were based on the cognitive representation.

\section{GENERAL DISCUSSION}

These experiments show that perception of a Roelofs effect is robust, being seen by all subjects under all delays. The Roelofs effect in visually guided behavior, though, depends much more strongly on the subjects and conditions. Half of the subjects showed a strong effect of a surrounding frame on pointing behavior, whereas the other half showed no effect. This bimodal distribution suggests that subjects went about the task in either of two different ways; the data are inconsistent with the hypothesis that subjects varied along a continuum of the amount of influence of the frame. Since all responses were made in a blank field after the stimuli had been extinguished, these differences may be due to differing strategies of the subjects; some responded in a motor mode, whereas others switched almost immediately to a cognitive mode, which brought the illusion along with it. All subjects appeared to operate in the cognitive mode when required to both point to and judge the perceived target location on each trial.

One need not assume that the two groups of subjects followed different psychological laws, but only that they switched from motor to cognitive modes at differing delays after stimulus offset. Discussion in terms of differential accuracy and decay rates of cognitive versus motor responses would redescribe the data, but would not explain their source.
All of the subjects showed a Roelofs effect in pointing when a long enough delay was interposed between target presentation and response or when pointing and judging occurred on the same trials; a closer titration of delay times would probably have shown a unique critical delay for a pointing Roelofs effect for each subject. Thus the results are not comparable to those from studies in which subjects respond while the stimuli are still present (Hansen \& Skavenski, 1977), or in which responses occur within $400-500 \mathrm{msec}$ of the target event (Hallett \& Lightstone, 1976; Wong \& Mack, 1981). All subjects in those studies showed accurate motor behavior, in the present interpretation because the spatial values in the motor representation had not yet been lost.

The appearance of the Roelofs effect with a delay between stimulus and motor response is reminiscent of the results of Wong and Mack (1981) that saccadic eye movements followed a veridical displacement direction with a short delay but followed a perceived displacement in the opposite direction after a longer delay. Though the delays used here were longer than those used by Wong and Mack, the pattern of results is similar to theirs. Thus it appears that if the motor representation of space possesses a memory for the positions of stimuli no longer present, the memory begins to degrade after no more than a few hundred milliseconds. The shift at a 4-sec delay in Experiment 1 puts an upper bound, but not a lower bound, on the memory buffer duration. The duration of this memory and the conditions under which it is degraded are topics for future research.

Other studies have provided data that can reinforce the contrast between cognitive and sensorimotor streams of visual information, though the studies were designed for other purposes and require interpretation if one is to relate them to the present framework. Abrams and Landgraf (1990) performed an induced-motion experiment with target and frame stimuli similar to ours and with two response measures. In one task, the subject pointed to the final position of the target. Extent of motion was determined on successive trials by pointing to the target in its left and right positions, respectively. In the other task, the subject moved a handle, similar to our pointer, through the same distance that the target was perceived to have moved. These tasks contrast with ours in that the finalposition pointing task (which they call a "location" condition) was egocentric, like our pointing task, but the motion reproduction task (which they call a "distance" condition) was exocentric; the subject indicated the perceived distance between two target positions, not the direction of either relative to the body. In both of our tasks, we took pains to obtain egocentric information. Our subjects responded to only one target at a time and never saw more than one target in the display, even during the training trials. In our theoretical framework, Abrams and Landgraf's location task probes the sensorimotor system, because that system's response is isomorphic to target position. The distance task probes the cognitive system, because the subject reproduces the perceived extent 
of movement. The pointer need not coincide with the target's final position.

Abrams and Landgraf's (1990) first experiment was analogous to our delay condition, and their second experiment was analogous to our no-delay condition-except that in both cases induced motion rather than Roelofs stimuli were used. Their results are consistent with what we found: with no delay, there was a significant interaction between the two tasks. There was an overall induced motion effect, but the significance of the effect was made difficult to interpret by the interaction, and it was not tested statistically for the two conditions separately. The extent of induced motion in their location condition, which we consider to be a sensorimotor measure, was only $0.5^{\circ}$ (estimated from their Figure 3 ). In the delay experiment, however, induced motion was seen with both measures, which is consistent with our delay condition in which Roelofs effects were seen for both cognitive and sensorimotor systems. There was no statistically significant interaction between their two measures in this experiment. Abrams and Landgraf's Experiment 3 showed, like our Experiment 3, that the results were not due to a straight-ahead shift.

Our results reinforce the conclusion that the normal human possesses two maps of visual space. One of them holds information used in perception: if a subject is asked what he or she sees, the information in this "cognitive" map is accessed. This map can achieve great sensitivity to small motions or translations of objects in the visual world by using relative motion or relative position as a cue. The price that the cognitive system pays for gaining this sensitivity is that it loses absolute egocentric calibration of visual space. A useful model for this process is the process of differentiation in calculus. In calculating motion $d x / d t$ by differentiation, the constant term (the spatial calibration) drops out of the representation.

The other visual map drives visually guided behavior, but its contents are not necessarily available to perception. This map does not have the resolution and sensitivity to fine-grained spatial relationships that the cognitive map has, but it is not required to: a small error in pointing, grasping, or looking is of little consequence. The advantage of this map is its robustness; the "motor" map is not subject to illusions such as induced motion and the Roelofs effect. It also has only a short memory, being concerned mainly with the here-and-now correspondence between visual information and motor behavior. If a subject must make motor responses to stimuli no longer present, this system must take its spatial information from the cognitive representation and brings any cognitively based illusions along with it. This is not to say that a sequence cannot be stored in memory and used to improve motor performance in the future; the current egocentric spatial values are lost, however. The relationships of information flow in the two systems are schematized in Figure 16.

Another way to interpret the relationship between cognitive and motor representations of visual space is to do

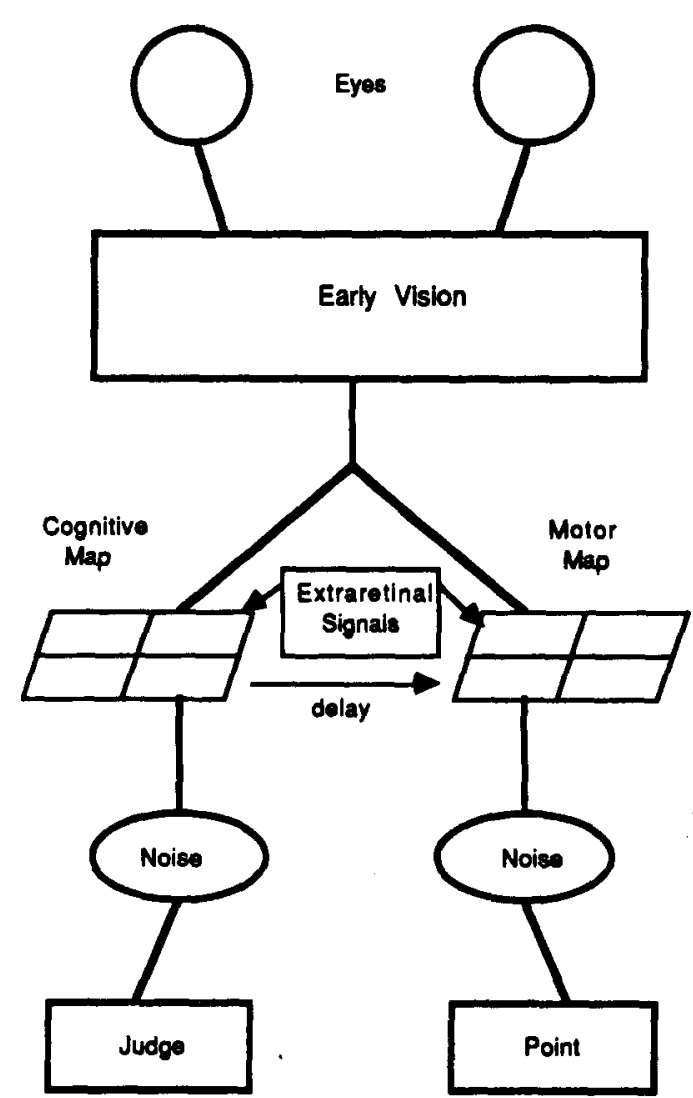

Figure 16. A proposed information flow scheme for perceptual judgments and visually guided behavior. After a common input stage, spatial information maps into both a cognitive representation (left center) and a motor representation (right center). Extraretinal information does not affect early vision, because receptive fields at the striate cortex and below have retinotopic rather than spatiotopic receptive field organization. The extraretinal information is supplied to the two maps separately because it may affect them differently. If the motor map has no spatial information, it can receive information from the cognitive map. The two maps influence behavior through independent noise sources.

so in terms of the ability to integrate information from each map with other information. The cognitive map's contents are coded in terms of the entire visual array (both target and frame in our case), whereas the motor map is generally inaccessible to integration with information from other sources. The difference between cognitive and motor representations in this context is analogous to the distinction between explicit and implicit modes of memory, respectively. The explicit mode is accessible to language and to experiential memory, whereas the implicit mode may hold information that the subject is unaware of or that is even contradictory to other explicit memory (Roediger, Weldon, \& Challis, 1988). Similarly, in our experiments, subjects could hold one position of a stimulus in the cognitive representation and simultaneously hold a different position for the same stimulus in the motor representation. 
Experiments done with stimuli with a potential confound between motion and position do not form the only basis for the two-visual-systems distinction; in others, clinical data have been used, as from blindsight, in which some visually guided motor capability remains despite lack of perception (Bridgeman \& Staggs, 1982; Weiskrantz, Warrington, Sanders, \& Marshall, 1974), or from other lesions (Goodale et al., 1994; Goodale, Pelisson, $\&$ Prablanc, 1986). We can conclude that the evidence for two distinct functional representations of visual space in humans is strong, but that the interaction between the systems is not as simple as it once seemed.

\section{REFERENCES}

Abrams, R. A., \& Landgraf, J. Z. (1990). Differential use of distance and location information for spatial localization. Perception \& Psychophysics, 47, 349-359.

Bacon, J. H., Gordon, A., \& Schulman, P. H. (1982). The effect of two types of induced-motion displays on perceived location of the induced target. Perception \& Psychophysics, 32, 353-359.

BRENNER, E., \& SMEETS, J. B. J. (1994). Different frames of reference for position and motion. Naturwissenschaften, 81, 30-32.

BRIDGEMAN, B. (1986). Multiple sources of outflow in processing spatial information. Acta Psychologica, 63, 35-48.

Bridgeman, B., Hendry, D., \& STARK, L. (1975). Failure to detect displacement of the visual world during saccadic eye movements. $V i$ sion Research, 15, 719-722.

Bridgeman, B., Kirch, M., \& Sperling, A. (1981). Segregation of cognitive and motor aspects of visual function using induced motion. Perception \& Psychophysics, 29, 336-342.

Bridgeman, B., \& Klassen, H. (1983). On the origin of stroboscopic induced motion. Perception \& Psychophysics, 34, 149-154.

Bridgeman, B., Lewis, S., Heit, G., \& Nagle, M. (1979). Relation between cognitive and motor-oriented systems of visual position perception. Journal of Experimental Psychology: Human Perception \& Performance, 5, 692-700

Bridgeman, B., \& Staggs, D. (1982). Plasticity in human blindsight. Vision Research, 22, 1199-1203.

Bridgeman, B., \& STARK, L. (1979). Omnidirectional increase in threshold for image shifts during saccadic eye movements. Perception \& Psychophysics, 25, 241-243.

Brosgole, L. (1968). An analysis of induced motion. Acta Psychologica, 28, 1-44.

Brune, F., \& Lücking, C. (1969). Oculomotorik, Bewegungswahrnehmung und Raumkonstanz der Sehdinge [The oculomotor system, motion perception and space constancy of visual objects]. Der Nerven$a r=t, 40,692-700$.

Dassonville, P., Schlag, J., \& Schlag-Rey, M. (1992). Oculomotor localization relies on a damped representation of saccadic eye displacement in human and nonhuman primates. Visual Neuroscience, 9, 261-269.

DitchBuRn, R. (1955). Eye-movements in relation to retinal action. $O p$ tica Acta, 1, 171-176

Felleman, D. J., \& Van Essen, D. C. (1991). Distributed hierarchical processing in the primate cerebral cortex. Cerebral Cortex, 1, 1-47.

FESTINGER, L., \& CANON, L. K. (1965). Information about spatial location based on knowledge about efference. Psychological Review, 72, 373-384

FOLEY, J. M., \& HELD, R. (1972). Visually directed pointing as a function of target distance, direction, and available cues. Perception \& Psychophysics, 12, 263-268.

Goodale, M. A., Meenan, J. P., Bulthoff, H. H., Nicolle, D. A., MurPhy, K. J., \& RACiCOT, C. I. (1994). Separate neural pathways for the visual analysis of object shape in perception and prehension. $C u r-$ rent Biology, 4, 604-610.

Goodale, M. A., Pelisson, D., \& Prablanc, C. (1986). Large adjustments in visually guided reaching do not depend on vision of the hand or perception of target displacement. Nature, 320, 748-750.

Hallett, P. E., \& Lightstone, A. D. (1976). Saccadic eye movements towards stimuli triggered during prior saccades. Vision Research, 16, 99-106.

HANSEN, R. (1979). Spatial localization during pursuit eye movements. Vision Research, 16, 1213-1221.

HANSEN, R., \& SKaVENSKI, A. (1977). Accuracy of eye-position information for motor control. Vision Research, 17, 919-926.

HoNDA, H. (1985). Spatial localization in saccade and pursuit-eyemovement conditions: A comparison of perceptual and motor measures. Perception \& Psychophysics, 38, 41-46.

HONDA, H. (1990). The extraretinal signal from the pursuit-eye-movement system: Its role in the perceptual and the egocentric localization systems. Perception \& Psychophysics, 48, 509-515.

MACK, A. (1970). An investigation of the relationship between eye and retinal image movement in the perception of movement. Perception \& Psychophysics, 8, 291-298.

Masson, G., Proteau, L., \& Mestre, D. R. (1995). Effects of stationary and moving textured backgrounds on the visuo-oculo-manual tracking in humans. Vision Research, 35, 837-852.

MateEFF, S., \& Gourevich, A. (1983). Peripheral vision and perceived visual direction. Biological Cybernetics, 49, 111-118.

PaILLARD, J. (1987). Cognitive versus sensorimotor encoding of spatial information. In P. Ellen \& C. Thinus-Blanc (Eds.), Cognitive processes and spatial orientation in animal and man (Vol. 2, pp. 43-77). Dordrecht: Martinus Nijhoff.

PaIllard, J. (1991). Motor and representational framing of space. In J. Paillard (Ed.), Brain and space (pp. 163-182). Oxford: Ox ford University Press.

Post, R. B., \& Leibowitz, H. W. (1985). A revised analysis of the role of efference in motion perception. Perception, 14, 631-643.

Prablanc, C., Echallier, J. F., Komilis, E., \& Jeannerod, M. (1979). Optimal response of eye and hand motor systems in pointing. Biological Cybernetics, 45, 113-124.

Roediger, H. L., III, Weldon, M., \& Challis, B. (1988). Explaining dissociations between implicit and explicit measures of retention: $\mathrm{A}$ processing account. In H. L. Roediger III \& F. I. M. Craik (Eds.), Varieties of memory and consciousness: Essays in honour of Endel Tulving (pp. 3-41). Hillsdale, NJ: Erlbaum.

Roelofs, C. (1935). Optische Localisation [Optical localization]. Archiv für Augenheilkunde, 109, 395-415.

SMEETS, J. B. J., \& BrenNer, E. (1995). Perception and action are based on the same visual information: Distinction between position and velocity. Journal of Experimental Psychology: Human Perception \& Performance, 21, 19-31.

Sugarman, R., \& Cohen, W. (1968). Perceived target displacement as a function of field movement and asymmetry. Perception \& Psychophysics, 3, 169-173.

WALLACH, H., \& LEWIS, C. (1965). The effect of abnormal displacement of the retinal image during eye movements. Perception \& Psychophysics, 1, 25-29.

Weiskrantz, L., Warrington, D., Sanders, M., \& Marshall, J. (1974). Visual capacity in the hemianopic field following restricted occipital ablation. Brain, 97, 709-729.

WERNER, H., WAPNER, S., \& BRUELL, J. (1953). Experiments on sensorytonic field theory of perception: VI. The effect of position of head, eyes, and of object on the position of the apparent median plane. Journal of Experimental Psychology, 46, 293-299.

WONG, E., \& MACK, A. (1981). Saccadic programming and perceived location. Acta Psychologica, 48, 123-131.

(Manuscript received June 2, 1995; revision accepted for publication May 20, 1996.) 